Test method

\title{
Development and application of micromechanical techniques for characterising interfacial shear strength in fibre-thermoplastic composites
}

\author{
L. Yang*, J.L. Thomason \\ University of Strathclyde, Department of Mechanical Engineering, 75 Montrose Street, Glasgow G1 1XJ, United Kingdom
}

\section{A R T I C L E I N F O}

\section{Article history:}

Received 20 May 2012

Accepted 2 July 2012

\section{Keywords:}

Micromechanical testing

Interfacial strength

Thermoplastic composites

\begin{abstract}
A B S T R A C T
The development of single fibre pull-out and microbond tests for characterising interfacial strength in thermoplastic composites is reviewed in detail. Manufacture of an experimental jig and sample preparation regimes for both tests are described. The challenges addressed in the sample preparation include the measurement of embedded fibre length for pull-out samples and the low yield rate of axisymmetric resin droplets obtained during sample preparation under nitrogen. The applications of these laboratory developed techniques are demonstrated by characterisation of the interfacial shear strength (IFSS) of glass fibre-polypropylene (GF-PP) and natural fibre-polylactic acid (NF-PLA). The comparison of the IFSS between neat and modified GF-PP showed that both methods were sensitive to the interfacial performance change despite the poor agreement between them for the absolute IFSS values from the same composite. The effect of the material modification was also reflected in load-displacement curves with different behaviour of the frictional motion after complete debonding. When a high level of fibre-matrix adhesion was realised in the composites with weak fibres, the microbond test showed higher feasibility for characterising the IFSS. This was clearly shown in its application to NF-PLA.
\end{abstract}

(c) 2012 Elsevier Ltd. All rights reserved.

\section{Introduction}

The fibre-matrix interface has been extensively studied due to its critical role in structural integrity and stress transfer in fibre reinforced composites. The ability to transfer the load across the interface requires the existence of fibre-matrix bonding. The origin of such bonding is usually discussed in terms of theories of adhesion with associated bonding mechanisms such as adsorption and wetting, electrostatic attraction, interdiffusion, chemical bonding and mechanical interlocking [1,2]. One of the generally accepted manifestations of adhesion is the mechanically measured value of interfacial shear strength

\footnotetext{
* Corresponding author. Tel.: +44 (0) 1415482324

E-mail address: l.yang@strath.ac.uk (L. Yang).
}

(IFSS), which has been widely used to characterise the adhesion in specific fibre-matrix systems.

Over the last four decades a number of experimental techniques have been developed to assess fibre-matrix interface bond quality by characterising IFSS either directly or indirectly. Reviews of these techniques and their related issues can be found in numerous books and papers [1,3-6]. It has become clear that micromechanical tests serve as a major tool for directly measuring interfacial tenacity in fibre reinforced composites. The test methods in this category include the single fibre pull-out test (SFPT), microbond test (MBT), fragmentation test and single fibre push-out test. The SFPT and MBT have been extensively studied since they were developed several decades ago $[7,8]$. In both tests, a fibre is partially embedded in a matrix, which is constrained while the fibre is loaded under tension. The external force applied to the fibre is recorded as a function of fibre end displacement during the 
debonding and subsequent pull-out process. In most cases, the embedded fibre length in the resin droplet in MBT is much smaller than the matrix block in SFPT. The essential difference between these two tests lies in their specimen scale and geometry. Some more recent development in micromechanical testing for IFSS focused on altering the sample geometry in MBT to reduce the data scatter [9-11].

Despite the fact that these test methods are commonly employed, there has been little, if any, standardisation of the sample preparation and test apparatus. Consequently, individual laboratories tend to create their own methods with essentially the same idea but different procedures. Although the application of MBT to thermosetting composites has attracted a great deal of attention over the years [4,12-15], it has been, to a lesser extent, considered for thermoplastic systems [16-18]. As a result, the procedure for using this method with thermoplastic polymers needs to be further improved. Moreover, the MBT may become very useful when a strong interfacial adhesion is achieved in thermoplastic composites through surface treatment or/and polymer modification.

The present work is aimed at giving details of the development of SFPT and MBT for fibre reinforced thermoplastic composites in our laboratory. It covers the manufacture of the experimental jig, sample preparation and mechanical testing analysis. The application of these techniques is demonstrated by characterising the interfacial shear strength (IFSS) of glass fibre-polypropylene (GFPP) and natural fibre-polylactic acid (NF-PLA). Results of the IFSS are analysed against the load-displacement behaviour and SEM photos of tested samples.

\section{Design and manufacture of the microvice}

In order to measure IFSS, shear stress needs to be generated across the interface during testing. This was achieved originally through a pair of shearing plates [8]. Such a configuration was adopted in this work and a microvice was designed and manufactured in the light of the work carried out by Craven and Viney [15]. Fig. 1 shows the model and the manufactured microvice. It consisted of a vice bottom, two vice tops and two vice ends. The vice tops could be assembled on to the vice bottom through a "dove-tail" joint and connected to the vice ends through a pair of springs at each end. The hole in the vice end was to assemble with a micrometer head and the holes in the vice top were fitted with nylon adjustment screws used to remove any twist in the vice. Both vice tops could be moved back and forth with a resolution of $1 \mu \mathrm{m}$ through a combination of a pair of springs and micrometer heads.

A pair of shearing plates were manufactured and assembled on the vice tops. Shearing plates play a critical role in the micromechanical test as they make direct contact with the matrix. Various configurations of shearing plates can be found in the literature [8,13-15]. We chose the configuration of a pair of shear plates with polished edges. Stainless steel with high carbon content was used to manufacture the plates to ensure a neat finish on the polished surface. The shearing plates were secured on the vice tops by a pair of metal bars as shown in Fig. 1. This arrangement allowed for flexible adjustment of the plates
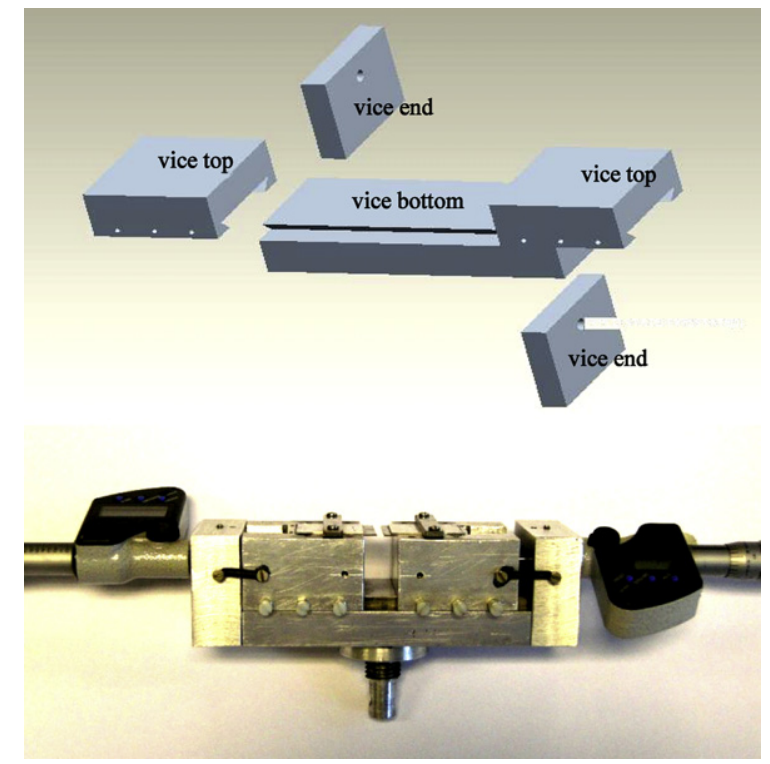

Fig. 1. Self-manufactured microvice for microbond and single fibre pull-out tests.

to eventually give a pair of thin parallel edges on the same plane.

\section{Sample preparation}

It is difficult to prepare single fibre-thermoplastic polymer samples for most micromechanical tests mainly because of their solid state at room temperature. Wetting of the thermoplastic polymers to the fibre normally has to be carried out at elevated temperature, at which the solid polymer becomes the viscous melt. In order to obtain consistent and repeatable results from a micromechanical test it is imperative that the techniques used to prepare samples for testing are consistent and identical for all samples created. The methods developed in this work are described as follows.

For the SFPT, the procedure to make samples is schematically illustrated in Fig. 2. The first step was to melt small pellets of the polypropylene (PP) on a glass slide using a hot plate heated to approximately $230{ }^{\circ} \mathrm{C}$. On melting of the pellets, another glass slide was positioned on top to create a sandwich configuration; thus creating a thin PP film between the slides. A $1 \mathrm{~kg}$ weight (also preheated on the hot plate) was then placed on top of the assembly to apply a holding pressure. This was then left on for $30 \mathrm{~s}$, after which the slides and PP film were removed from the hot plate and placed on a glass block to cool down to room temperature. On cooling, the PP film was removed from the slide and the thickness of the film was measured. The film was then cut with razor blades into a square $(13 \times 13 \mathrm{~mm})$. The next stage of the process was to sandwich the glass fibres between a set of the square PP films. This was achieved by laying down one of the films onto a glass slide; the glass fibres were then positioned across the film and secured at either end of the slide by adhesive tape. Another PP film was then positioned on top of the fibres, square to 


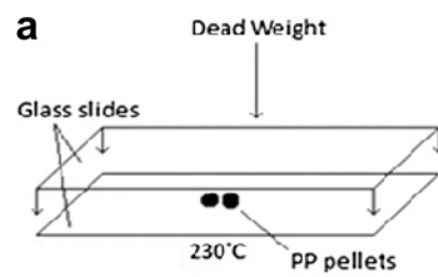

d

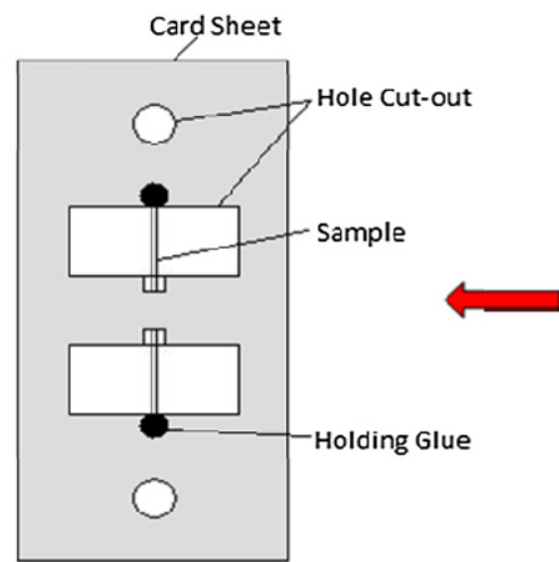

b

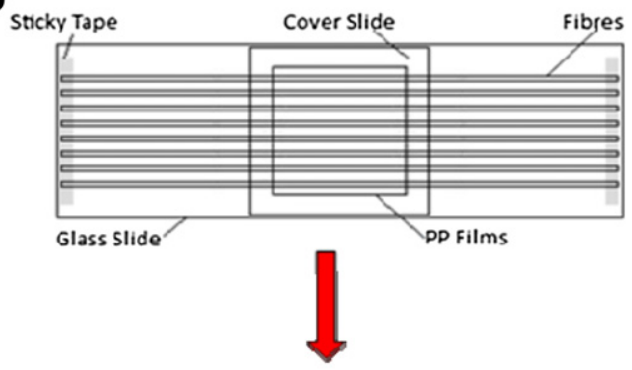

C

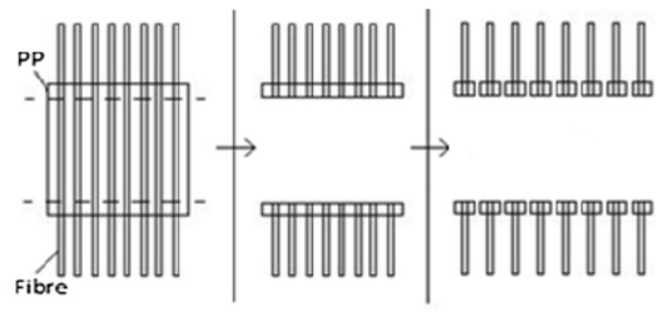

Fig. 2. Schematic illustration of sample preparation for the single fibre pull-out.

the film below, thus creating a PP film and glass fibre sandwich assembly. A small glass cover slide was then positioned over the PP films in order to reduce oxidisation during the next heating stage. The next process was to heat the assembly in order to melt the PP films and thus embed the fibres. This was achieved through placing the assembly in a Mettler FP82 hot stage at $220^{\circ} \mathrm{C}$ for $4 \mathrm{~min}$, after which the sample was removed and cooled at ambient room temperature. The PP film (and embedded fibres) was then removed from the glass slide and dissected into individual samples as shown in Fig. 2. Care had to be taken when cutting up the samples to ensure that the length of embedded fibre left after cutting was not too long, as the embedded length $\mathrm{L}_{\mathrm{e}}$ is limited by the strength and diameter of the fibre, $\sigma_{\mathrm{fu}}$ and $\mathrm{D}_{\mathrm{f}}$, respectively and the IFSS, $\tau_{\mathrm{ult}}$; according to Eq (1)

$L_{e}<\frac{\sigma_{f u} D_{f}}{4 \tau_{u l t}}$

It was also important to vary the embedded length to obtain a wide range of embedded areas to test. With the samples mounted on the card as shown in Fig. 2, it was then possible to measure the fibre diameter of each sample using a microscope. Measurement of $\mathrm{L}_{\mathrm{e}}$ prior to the test may be subject to significant error due to the curved surface on the bottom and top of the sample as shown in Fig. 3. $\mathrm{L}_{\mathrm{e}}$ measured from the boundary of the matrix is larger than the true value in this case. Moreover, it is seen that in the zero-gap loading mode the plates do not act directly at the meniscus as expected but on the matrix outer region instead. As a result, the sample may gradually deviate from the loading direction and even completely flip over during the test. The results obtained from such samples were discarded in this work. Furthermore, the uneven top surface of the sample may lead to a bending configuration when it makes contact with the plates.

For the MBT, the method developed previously proved quite efficient when forming PP microdroplets on a single fibre in air $[19,20]$ and gave a yield rate above $95 \%$. However, it was then found that severe thermo-oxidative degradation occurred to resin microdroplets in the period of heating and caused significant decrease in the measured IFSS of GF-PP [21]. Consequently, it was suggested that the samples be made under inert gas such as nitrogen. Although the use of a nitrogen successfully prevented polymer droplets from any degradation, it also led to an extremely low yield rate $(<10 \%)$ of the axisymmetric resin microdroplets required for the MBT. This was the case even after holding the samples at
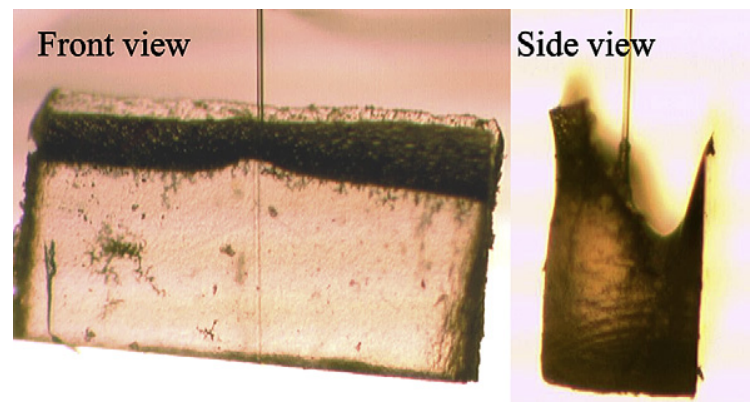

Fig. 3. Stereomicroscopy view of a typical single fibre pull-out sample. 
$220{ }^{\circ} \mathrm{C}$ for a prolonged period of time. An example of such non-axisymmetric droplet is shown in Fig. 4. It was realised that after the first a few minutes of heating there was actually no further change in the yield rate by extending the length of this process. In other words, the yield rate was independent of the period of heating after just several minutes. On the other hand, it has been reported in the Wilhelmy balance method that, due to the low surface tension of neat PP melt, the complete wetting of the bare GF surface is thermodynamically favourable at $230{ }^{\circ} \mathrm{C}[22,23]$. Therefore, the explanation for the low yield rate is most likely to be associated with the viscosity of the polymer melt, which is controlled by the temperature. For a non-axisymmetric droplet on a cylindrical fibre, the part that has excessive melt is expected to be under a higher internal pressure than the part that has less melt due to the different curvature of the surface. The difference of this internal pressure serves as a driving force in forming a symmetric droplet around the fibre. However, when the melt viscosity is too high this force may not be sufficient to efficiently move the melt in a reasonable length of time. In the case of an air atmosphere the occurrence of thermo-oxidative degradation of PP can reduce the melt viscosity through chain scission, which effectively reduces the average molecular weight. As a result, a high yield rate of the microbond samples can be obtained when they are made in air. It should be noted that the MFI of PP used in this study is actually quite high and yet the melt viscosity seems to be high enough to overcome the internal pressure. Another observation from some non-axisymmetric droplets was incomplete inclusion of the fibre in the matrix, as shown in Fig. 4. This inhibited the development of full contact line at both ends of the droplet and, in turn, the droplet formation. In order to solve these problems, a PP fibre was tied around the glass fibre prior to heating so that the PP distributed more evenly around the fibre and the full contact line readily formed during melting. The capillary force then elongated the droplet and formed the meniscus. The formation of axisymmetric microdroplets was, therefore, expected to be facilitated. Indeed, the experiment showed that a high yield rate of $95 \%$ was obtained for the microbond samples using this method.

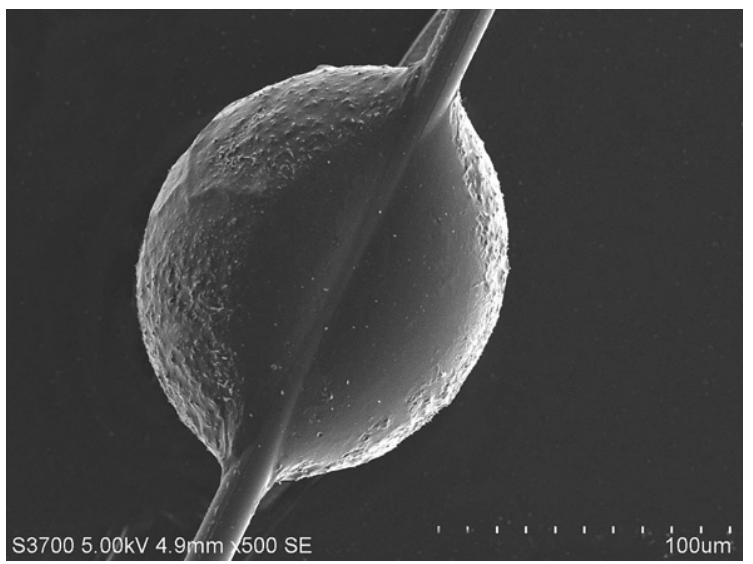

Fig. 4. SEM photo of a non-axisymmetric PP microdroplet on a single glass fibre.

\section{Single fibre pull-out test (SFPT) and microbond test (MBT)}

The SFPT and the MBT were carried out in the same experimental jig. To perform the fibre pull-out test, the sheets of card were cut to create a tab with the sample hanging off. A steel hook attached to a $10 \mathrm{~N}$ load cell on an Instron Model 3342 tensile testing machine was positioned through the circular cut-out section to act as the loading point. In order to aid the setting up of testing and then be able to observe the debonding and pull-out process, a stereo microscope (45 $\times$ magnification) with a digital camera attached - feeding real time video to a computer screen was placed in front of the test area. The microscope made it possible to accurately position the sample and observe the fibre pull-out during testing. Due to the small size of resin microdroplets in MBT, some card frame was left taped to the bottom of the fibre to keep it under tension $(<0.5 \mathrm{mN})$ so that the sample could be perfectly aligned with the loading direction prior to testing. This was not necessary for the specimens in SFPT due to the higher mass of polymer supported by the test fibre. The free fibre length was kept constant at $5 \mathrm{~mm}$ throughout all the tests. The loading edges of the shearing plates were brought to make contact with the fibre in both methods. With the sample in place the test could then start and the load cell extended the fibre at a constant rate of $0.1 \mathrm{~mm} / \mathrm{min}$. Fig. 5 shows the samples engaged in testing. Pull-out of the fibre can be clearly observed in SFPT after complete interfacial debonding. It is also seen in Fig. 5 that the optical reflection of the sample in the image can actually be used to monitor the misalignment of the fibre axis with the loading direction by characterising the angle between the fibre in the reflection and the real fibre below the plates. The misalignment was found to be less than $5^{\circ}$ in the MBT and less than $20^{\circ}$ in the SFPT. The larger misalignment in the latter case was due to the rough contact surface of the matrix. During the test, a load versus displacement plot was produced in real time. Observation of these real-time plots helped to identify when debonding had occurred and also when the fibre was completely pulled out. In both methods, the test plot (although they can vary in shape significantly in some cases) exhibited an initial ramp representing the sample being loaded as the load cell increased the extension, followed by a peak load, $\mathrm{F}_{\max }$, where it was thought that the complete debonding between the fibre and the matrix occurred. After $F_{\max }$, the shape of the plot could vary significantly with the specific system, as discussed in the next section.

After testing, the overall interfacial shear strength, $\tau_{\text {app}}$, can be calculated by

$\tau_{\text {app }}=\frac{F_{\max }}{\pi D_{f} L_{e}}$

where $D_{f}$ and $L_{e}$ are the fibre diameter and the embedded fibre length measured for each individual sample. Both parameters were measured using a Nikon Epiphot inverted optical microscope. $\mathrm{L}_{\mathrm{e}}$ of the MBT sample was the distance between two meniscuses, and for the SFPT sample it was the length from the sample bottom line to the middle line of the black area, as seen in Fig. 3. IFSS can be characterised 

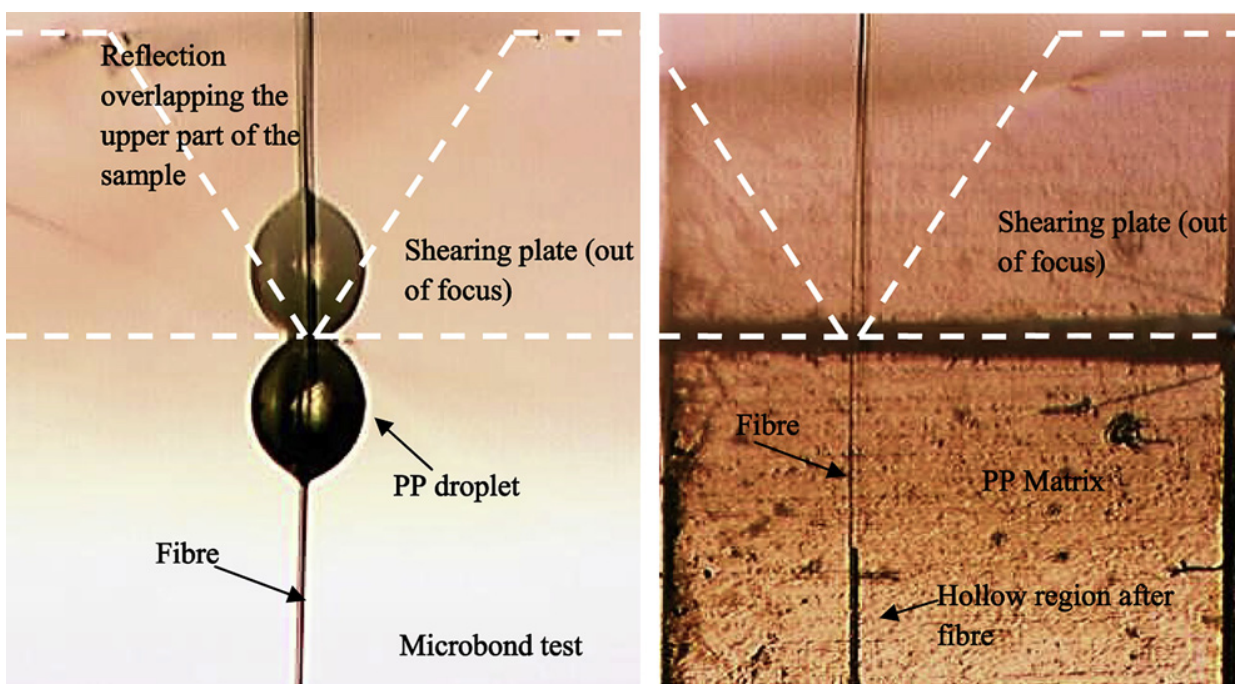

Fig. 5. Stereomicroscopy view of microbond and single fibre pull-out samples during testing

by $\tau_{\text {app }}$ when the interfacial shear stress is constant over $\mathrm{L}_{\mathrm{e}}$, and the entire interface debonds simultaneously. However, this is not usually the case since the stress is often concentrated in the interface region closest to the loading point. Nevertheless, there are at least two scenarios where $\tau_{\text {app }}$ may be valid for characterisation of the IFSS: 1 ) the interface fails by yielding, which evens out the stress at the interface at debonding and 2) the frictional stress in the debonded area is much greater than the local interfacial bonding strength. Despite this complexity in the choice of the characteristic parameter, it seems that $\tau_{\mathrm{app}}$ is normally sensitive enough to distinguish the level of fibre-matrix adhesion in a comparative study and correlate well with the change in the macroscopic properties of the composites with various kinds of interfacial modification [22,24,25].

The maximum error $\delta \tau_{\text {app }}$ of $\tau_{\text {app }}$ can be expressed by

$\left|\frac{\delta \tau_{\text {app }}}{\tau_{\text {app }}}\right|=\left|\frac{\delta F_{\max }}{F_{\max }}\right|+\left|\frac{\delta D_{f}}{D_{f}}\right|+\left|\frac{\delta L_{e}}{L_{e}}\right|$

where $\delta F_{\max }, \delta \mathrm{D}_{\mathrm{f}}$, and $\delta \mathrm{L}_{\mathrm{e}}$ are the errors of the corresponding parameters. The major component of this error may come from the measurement of $\mathrm{L}_{\mathrm{e}}$ because of the meniscus in the microbond sample and curved surface in the pull-out sample mentioned earlier. Nevertheless, it was found that only a small part of the meniscus was left behind on the fibre if the polymer was not degraded in the microbond sample, and the effect of curved surface could also be significantly reduced by maintaining a fairly large $\mathrm{L}_{\mathrm{e}}$ and small film thickness in the pull-out sample. Alternatively, $\mathrm{L}_{\mathrm{e}}$ of pull-out samples may be obtained from the test plot. However, this would give reasonable values only when the load caused by dynamic frictional stress after debonding is very close to $F_{\max }$.

\section{Characterisation of IFSS in fibre-reinforced thermoplastic composites}

\subsection{Glass fibre-polypropylene (GF-PP)}

The materials used to prepare GF-PP samples included the bare E-glass fibres supplied by Owens Corning Vetrotex and the sized E-glass fibres $\left({ }^{\circledR}\right.$ TufRov 4575$)$ provided by PPG Industries. The latter had been treated with a proprietary PP-compatible size and resulted in a heterogeneous surface compared to the featureless surface of the bare fibre, as shown in Fig. 6. Both types of fibre had a similar

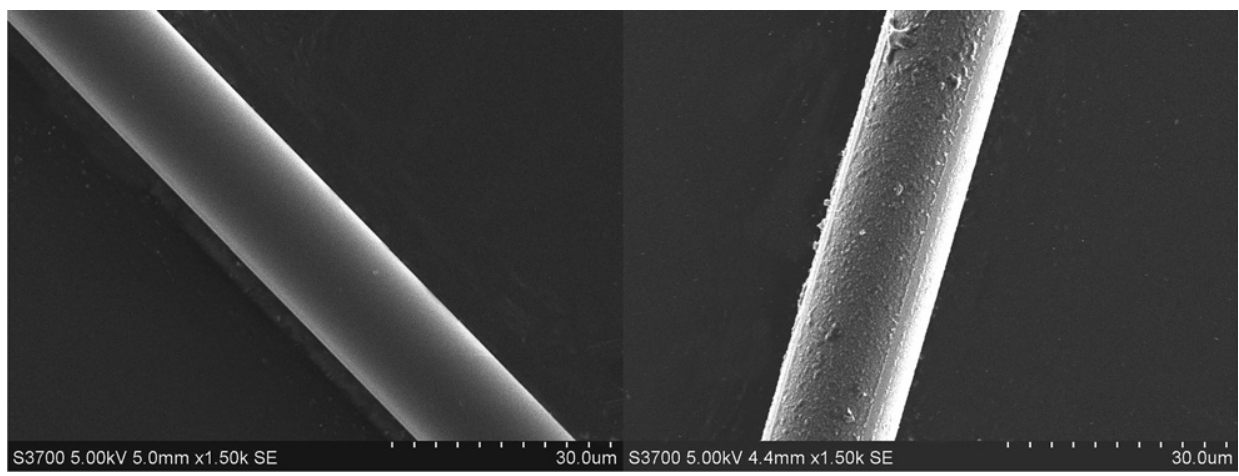

Fig. 6. SEM photos of bare glass fibre (left) and sized glass fibre (right). 
Table 1

Summary of results for IFSS of GF-PP measured by both SFPT and MBT.

\begin{tabular}{lllr}
\hline Fibre-matrix system & Testing method & Number of the sample & Embedded length $(\mu \mathrm{m})$ \\
\hline BGF-PPh & MBT & 17 & $123-344$ \\
SGF-PPh & MBT & 16 & $200-330$ \\
SGF-PPh & SFPT & 16 & $180-994$ \\
SGF-PPm & MBT & 21 & $16.4 \pm 1.8$ \\
SGF-PPm & SFPT & 36 & 2.5 \\
\hline
\end{tabular}

average diameter of $17.5 \mu \mathrm{m}$. The polymer matrices used in this work were supplied by PPG industries including homopolymer PP (PPh) and blends (PPm) of PPh combined with $2 \mathrm{wt} \%$ maleic anhydride grafted PP (MaPP). The PP in these two matrices had a MFI of $120 \mathrm{~g} / 10 \mathrm{~min}$ at $230^{\circ} \mathrm{C}$ and $2.16 \mathrm{~kg}$.

The results for the measured IFSS in GF-PP are summarised in Table 1. It can be seen from the MBT results for SGF-PPh that the coating on the fibre alone already led to a significant increase in the IFSS compared to BGF-PPh. Further improvement in IFSS was obtained by the addition of a small amount of MaPP into PPh. The modified system, SGF-PPm, gave a value of the IFSS more than twice the value obtained with the unmodified BGF-PPh. A similar trend was found in the SFTP results, showing a considerable increase of the IFSS resulting from the combination of surface coating on the glass fibre and addition of MaPP to $\mathrm{PPh}$. It is also noticeable that, for the same system, the value of the IFSS measured by the SFTP is much smaller than that obtained by the MBT. Particularly for SGF-PPh, the IFSS obtained from SFPT is even smaller than that obtained from the MBT for BGF-PPh. It is clear that there is only limited compatibility between these two customised methods in this case. However, we previously reported good agreement between these methods on the measured IFSS of bare glass fibre-polypropylene with a higher molecular weight [26]. Apart from the materials tested, another difference is the PP film thickness, which was 0.1$0.15 \mathrm{~mm}$ in the last study and $0.3-0.5 \mathrm{~mm}$ in this one Thicker samples not only lead to a greater uncertainty of the fibre embedded length, as mentioned earlier, but also create a bumpier surface. When the shearing plates compress the sample, a bending configuration may be developed. This can result in a tensile stress at the interface and cause premature debonding. The failure may be further facilitated by misalignment between the embedded fibre and the loading direction. Moreover, cutting at the bottom of the sample may also cause damage to the interface, especially for shorter embedded lengths. More pull-out samples with much smaller $\mathrm{L}_{\mathrm{e}}$ were used in this work due to the high IFSS in modified GF-PP reducing the maximum allowable value of $\mathrm{L}_{\mathrm{e}}$.

It is very interesting to observe the different behaviour exhibited in the course of fibre being pulled out of the matrix after complete debonding $\left(\mathrm{F}_{\max }\right)$. The behaviour was found to coincide with different levels of IFSS measured in GF-PP. Fig. 7 presents some examples of the loaddisplacement curve from the SFPT. For IFSS approximately below $5 \mathrm{MPa}$, there appears to be a monotonic decrease of the load after the sharp drop at $\mathrm{F}_{\max }$ as shown in Fig. 7(a). As IFSS increases above $5 \mathrm{MPa}$, a stick-slip effect can be observed. The amplitude of the sudden load drops and the frequency of stick-slip oscillation are also related to the measured IFSS. Lower IFSS tends to generate a combination
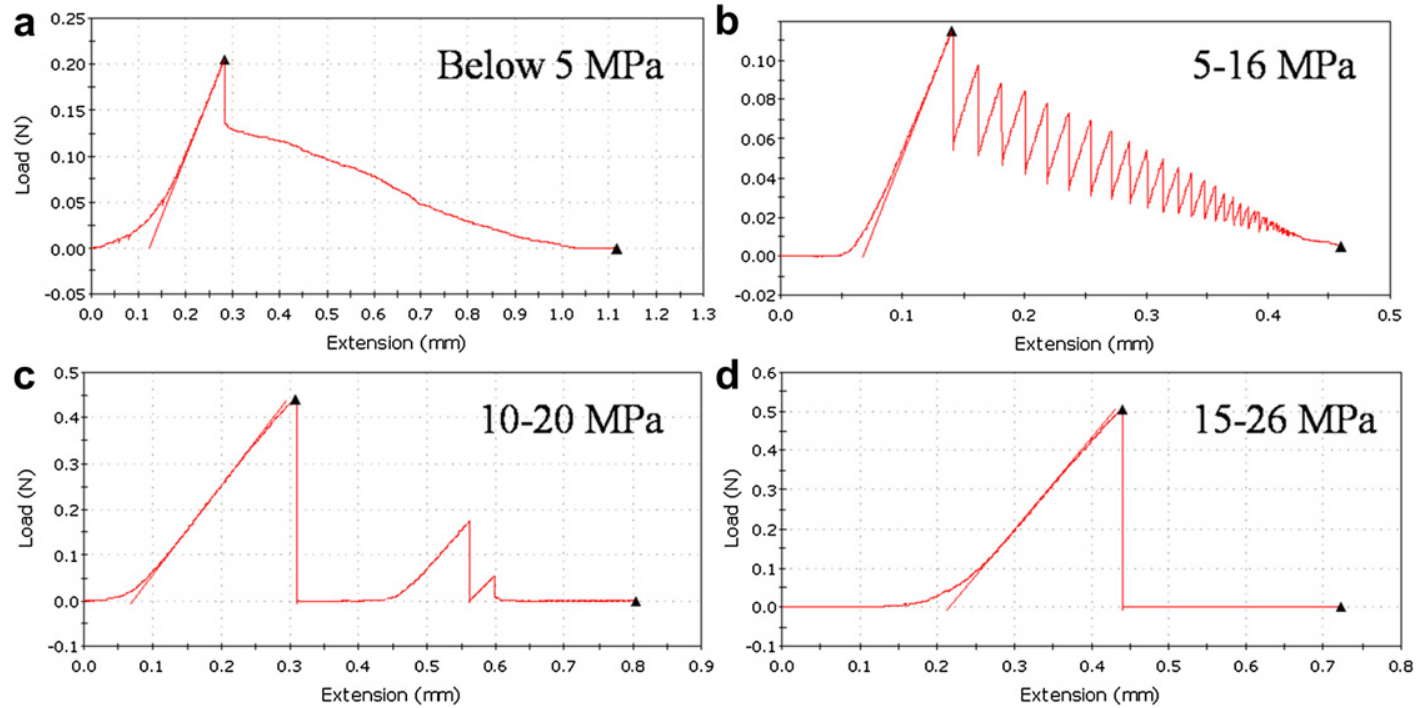

Fig. 7. Different behaviours of load-displacement curves obtained from single fibre pull-out test on GF-PP. 


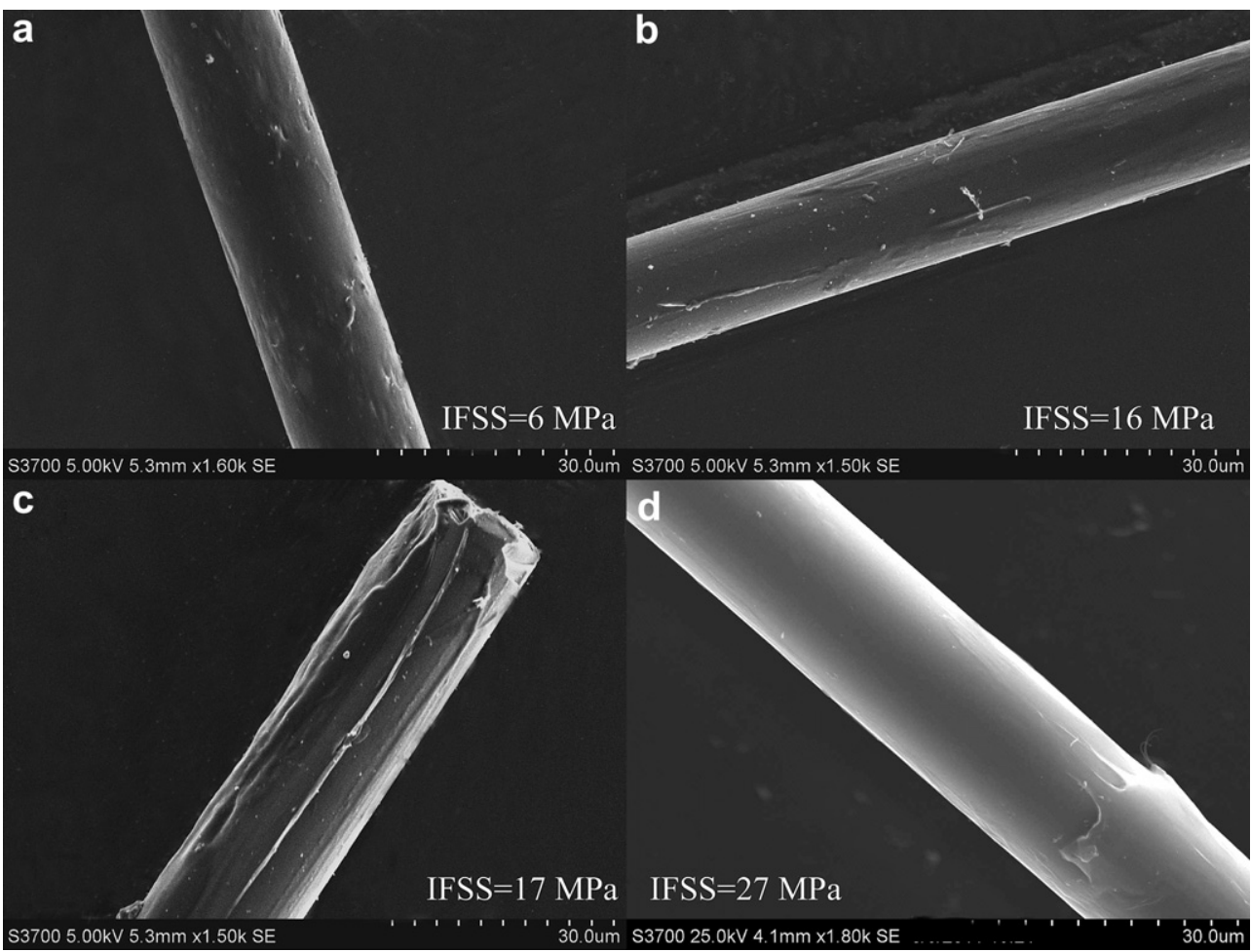

Fig. 8. SEM photos of several pulled-out sized glass fibres.

of low amplitude and high frequency, as shown in Fig. 7(b), whereas higher IFSS usually corresponds to the oscillation with high amplitude and low frequency, as shown in Fig. 7(c). When the maximum force is very high, the sudden release of the stored energy can result in complete loss of the measured force by the 'jump' of a large portion of the embedded fibre. This can sometimes produce fibre slack, which leads to the subsequent displacement at no registered force until the fibre is restrained again, as shown in Fig. 7(c). In extreme cases, the entire fibre can jump out of the matrix directly after debonding, as shown in Fig. 7(d). It is seen that the different stick-slip motions shown in Fig. 7(b-d) are not found in an IFSS range independent of each other, but instead these ranges can overlap somewhat. Furthermore, it can be seen from Fig. 7(d) that the loaddisplacement curve can no longer be used in this case to distinguish the occurrence of fibre breakage from interfacial failure. A post-test microscopy examination is, therefore, required of the tested PP film to confirm interfacial failure. The stick-slip motion was also observed in the MBT (not shown here) and further discussion on the stick-slip effect in both SFPT and MBT will be carried out in the future.

Fig. 8 presents some SEM micrographs of the debonded area of sized fibres after testing. Interpreting useful information of the failure mode from the debonded area is a little uncertain when a sized fibre has nonuniformly distributed coating, as shown in Fig. 6. A number of SEM photos (some shown in Fig. 8) seem to give an impression that most sized fibres after testing had a trace of residual material on the debonded area. The residual material could be part of the fibre-matrix interphase and fully/partially cover the fibre embedded area. It was inconclusive as to whether there was any correlation between the observation of the debonded area and the measured IFSS for the modified systems. Nevertheless, according to the SEM results it may be reasonable to state that most samples tested by the methods developed in this work failed at the interface between the fibre surface and the interphase or within the fibre-matrix interphase.

\subsection{Natural fibre-polylactic acid}

Two types of natural fibres (unretted flax and hemp) and the biodegradable polylactic acid (PLA) were received from Sirris Leuven Composites Application Lab (SLC Lab). The polymer had a MFI of $4 \mathrm{~g} / 10 \mathrm{~min}$ at $210{ }^{\circ} \mathrm{C}$ and $2.16 \mathrm{~kg}$. The single fibres that were extracted from the fibre strand had a diameter range from $17 \mu \mathrm{m}$ to $120 \mu \mathrm{m}$. The microbond samples were fabricated by the procedure described earlier for GF-PP except that the samples were held at $200{ }^{\circ} \mathrm{C}$ in air for $10 \mathrm{~min}$.

The commonly used natural fibres in polymer composites have a tensile strength less than $1 \mathrm{GPa}[27,28]$ which can be further significantly reduced at elevated temperature due to their poor thermal stability [29,30]. If such fibres can form a very strong interfacial bond with the matrix, the maximum embedded fibre length for a successful interfacial debonding will be considerably limited. This was exactly the case in the present work, where both Flax-PLA and Hemp-PLA gave the IFSS above $20 \mathrm{MPa}$. The fibre embedded length in these samples had to be kept below $200 \mu \mathrm{m}$ to offer a good chance 

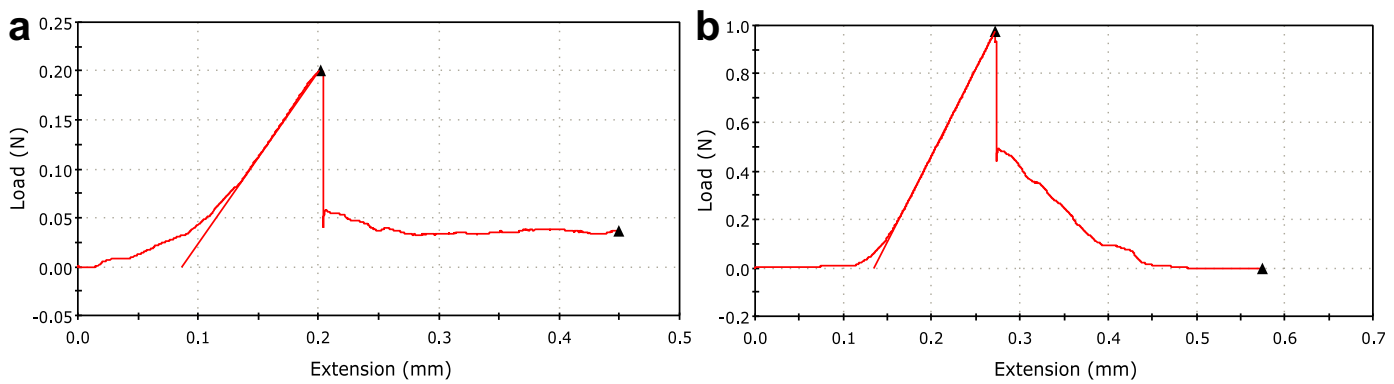

Fig. 9. Load-displacement cures obtained from the microbond test on the flax fibre without (a) and with (b) significant diameter variation immediate below the resin microdroplet.

for the interfacial debonding to occur before fibre breaking. As a consequence, only the microbond method was used in this case. The diameter variation in a natural fibre might be negligible within a small embedded fibre length. However, it was found that diameter change along the fibre could strongly influence the load-displacement curve after debonding, as shown in Fig. 9. A typical constant load trace was observed in Fig. 9(a) after the interfacial failure when there was no significant change in the diameter of the fibre below the resin droplet. However, a load-displacement curve can sometimes appear to be analogous to that in the single fibre pull-out test, as seen in Fig. 9(b), if the fibre diameter dramatically decreased below the droplet as, shown in Fig. 10.

It should be emphasised that the PLA microdroplets were formed at elevated temperature in air and they may undergo thermo-oxidative degradation, as found to be the case for GF-PP microbond samples [26]. In order to confirm this, the dimension change in the polymer microdroplets was examined using hot-stage microscopy. Fig. 11 shows a change of the maximum droplet diameter as a function of processing time under thermal conditions used in the sample preparation for a PLA droplet with an initial diameter of $175 \mu \mathrm{m}$. It can be clearly seen that the droplet diameter decreases linearly with the time during the observation. There is a great difference in the rate of diameter reduction between PLA and PP microdroplets. For a PP droplet formed at $220{ }^{\circ} \mathrm{C}$ in air, the rate of diameter reduction was found to be approximately $7.5 \mu \mathrm{m} / \mathrm{min}$ when the initial droplet diameter was $175 \mu \mathrm{m}$ [26], while it is only $0.4 \mu \mathrm{m} / \mathrm{min}$ for PLA droplet formed at $200{ }^{\circ} \mathrm{C}$ in air.

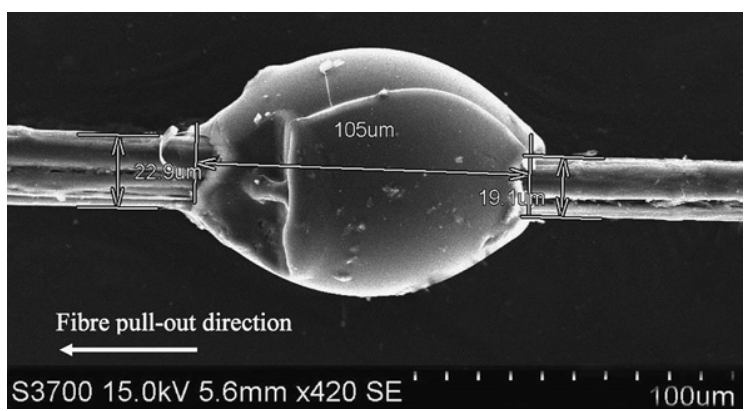

Fig. 10. SEM photo of a tested microbond sample of Flax-Polylactic acid.

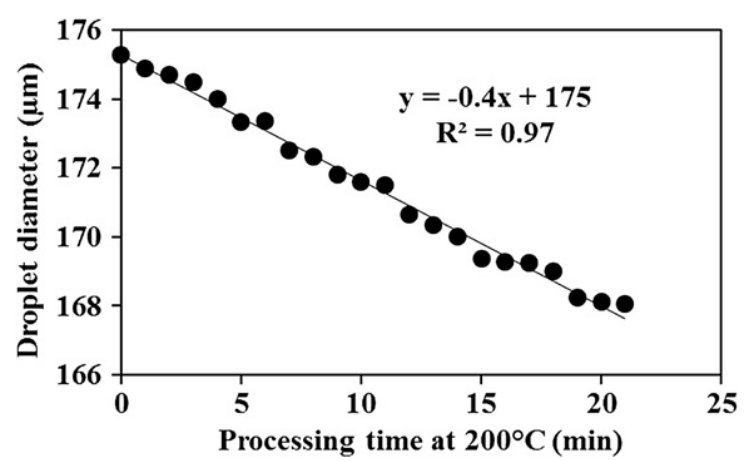

Fig. 11. Diameter reduction of PLA microdroplet as a function of processing time at $200{ }^{\circ} \mathrm{C}$ in air.

Nevertheless, it is evident that NF-PLA samples might experience some level of polymer degradation during the sample preparation.

\section{Conclusions}

Customised microbond and single fibre pull-out tests were developed in this work to characterise the interfacial shear strength of fibre reinforced thermoplastic polymers. Detailed description was given of manufacturing the experimental jig, sample preparation, and micromechanical testing. It was emphasised that sample preparation could be complex with thermoplastic polymers, whose thermal stability must be considered under any chosen heating programme. The yield rate of resin microdroplets on a single fibre was greatly improved by tying a glass fibre with a polymer fibre. The developed techniques were applied to glass fibre-polypropylene composites and the resolution was demonstrated to be sufficient to clearly distinguish effects of surface treatment of the glass fibre and matrix modification on the fibre-matrix adhesion. The results obtained from both methods agreed that a significant increase in the IFSS of GF-PP can be achieved through fibre sizing and addition of MaPP into PP. Moreover, different levels of IFSS were also reflected in the loaddisplacement curves in the single fibre pull-out test. Low IFSS corresponded to a monotonic decrease in the load after complete debonding while high IFSS correlated with stickslip motion. Inspection of the debonded area of sized glass 
fibres seemed to suggest that GF-PP samples in these tests failed at the interface between the fibre and the interphase or within the fibre-matrix interphase. Despite the fact that a similar trend was captured by both methods for the change of IFSS in different systems, the agreement between MBT and SFPT was quite poor for the same system. The application of the microbond technique to natural fibrepolylactic acid showed the ability of this method to deal with the system combining weak fibres with strong fibrematrix adhesion. It was also found that a significant change in fibre diameter could highly influence the loaddisplacement curve after debonding.

\section{Acknowledgements}

The authors gratefully acknowledge the financial support from Glasgow Research Partnership in Engineering (GRPE), PPG Industries, and "Nature Wins" Project (IWT 100385). The authors would also like to thank PPG Industries, Owens Corning Vetrotex, and Sirris and SLC-Lab (Belgium) for providing the materials used in this study. Special thanks are given to Advanced Materials Research Laboratory (AMRL) for the use of SEM in this work.

\section{References}

[1] J. Kim, Y. Mai, Engineered Interfaces in Fiber Reinforced Composites, Elsevier Science Ltd, Oxford, 1998.

[2] D. Hull, T.W. Clyne, An Introduction to Composite Materials, second ed., Cambridge University Press, 1996.

[3] F.R. Jones, Handbook of Polymer-fibre Composites, Longman Scientific and Technical, Essex and New York, 1994

[4] P.J. Herrera-Franco, L.T. Drzal, Comparison of methods for the measurement of fibre/matrix adhesion in composites, Composites 23 (1992) 2-27.

[5] S. Zhandarov, E. Mäder, Characterization of fiber/matrix interface strength: applicability of different tests, approaches and parameters, Composites Science and Technology 65 (2005) 149-160.

[6] R.J. Gray, Experimental techniques for measuring fibre/matrix interfacial bond shear strength, International Journal of Adhesion and Adhesives 3 (1983) 197-202.

[7] L.J. Broutman, Measurement of the Fibre-polymer Matrix Interfacial Strength, Interfaces in Composites, ASTM STP 452, 1969, pp. 27-41.

[8] B. Miller, P. Muri, L. Rebenfeld, A microbond method for determination of the shear strength of a fiber/resin interface, Composites Science and Technology 28 (1987) 17-32.

[9] N.-S. Choi, J.-E. Park, Fiber/matrix interfacial shear strength measured by a quasi-disk microbond specimen, Composites Science and Technology 69 (2009) 1615-1622.

[10] Z. Liu, X. Yuan, A.J. Beck, F.R. Jones, Analysis of a modified microbond test for the measurement of interfacial shear strength of an aqueous-based adhesive and a polyamide fibre, Composites Science and Technology 71 (2011) 1529-1534.

[11] B. Morlin, T. Czigany, Cylinder test: development of a new microbond method, Polymer Testing 31 (2012) 164-170.

[12] R.J. Day, J.V.C. Rodrigez, Investigation of the micromechanics of the microbond test, Composites Science and Technology 58 (1998) 907-914.
[13] H.D. Wagner, H.E. Gallis, E. Wiesel, Study of the interface in Kevlar 49-epoxy composites by means of microbond and fragmentation tests: effects of materials and testing variables, Journal of Materials Science 28 (1993) 2238-2244

[14] A. Hodzic, S. Kalyanasundaram, A. Lowe, Z.H. Stachurski, The microdroplet test: experimental and finite element analysis of the dependence of failure mode on droplet shape, Composite Interfaces 6 (1998) 375-389.

[15] R.C.J.P. Craven, C. Viney, Evaluating the silk/epoxy interface by means of the microbond test, Composites: Part A 31 (2000) 653-660.

[16] R.-B. Adusumalli, H.K. Weber, T. Roeder, H. Sixta, W. Gindl, Evaluation of experimental parameters in the microbond test with regard to Lyocell fibers, Journal of Reinforced Plastics and Composites 29 (2010) 2356-2367.

[17] L. Gonon, A. Momtaz, D. Van Hoyweghen, B. Chabert, J.F. Gérard, R. Gaertner, Physico-chemical and micromechanical analysis of the interface in a poly(phenylene sulfide)/glass fiber composite-a microbond study, Polymer Composites 17 (1996) 265-274.

[18] A. Le Duigou, P. Davies, C. Baley, Interfacial bonding of flax fibre/ poly(L-lactide) bio-composites, Composites Science and Technology 70 (2010) 231-239.

[19] L. Yang, J.L. Thomason, Interface strength in glass fibrepolypropylene measured using the fibre pull-out and microbond methods, Composites Part A: Applied Science and Manufacturing 41 (2010) 1077-1083.

[20] L. Yang, J.L. Thomason, Fibre-polymer Interface Adhesion-the Microbond Test. in (2010), http://www.strath.ac.uk/ compositematerials/videos/.

[21] L. Yang, J.L. Thomason, W. Zhu, The influence of thermo-oxidative degradation on the measured interface strength of glass fibrepolypropylene, Composites Part A: Applied Science and Manufacturing 42 (2011) 1293-1300.

[22] E. Mäder, H.J. Jacobasch, K. Grundke, T. Gietzelt, Influence of an optimized interphase on the properties of polypropylene/glass fibre composites, Composites Part A: Applied Science and Manufacturing 27 (1996) 907-912.

[23] P. Nygard, K. Grundke, E. Mader, C. Bellmann, Wetting kinetics and adhesion strength between polypropylene melt and glass fibre: influence of chemical reactivity and fibre roughness, Journal of Adhesion Science and Technology 16 (2002) 1781-1808.

[24] E. Mäder, E. Moos, J. Karger-Kocsis, Role of film formers in glass fibre reinforced polypropylene - new insights and relation to mechanical properties, Composites Part A: Applied Science and Manufacturing 32 (2001) 631-639.

[25] J.L. Thomason, G.E. Schoolenberg, An investigation of glass fibre/ polypropylene interface strength and its effect on composite properties, Composites 25 (1994) 197-203.

[26] L. Yang, J.L. Thomason, The influence of oxidative-thermal degradation of polypropylene on measured interface strength of glass fibre-polypropylene, in: 14th European Conference On Composite Materials, Budapest, Hungary, 2010.

[27] M.C. Symington, W.M. Banks, O.D. West, R.A. Pethrick, Tensile testing of cellulose based natural fibers for structural composite applications, Journal of Composite Materials 43 (2009) 10831108.

[28] J.L. Thomason, J. Carruthers, J. Kelly, G. Johnson, Fibre cross-section determination and variability in sisal and flax and its effects on fibre performance characterisation, Composites Science and Technology 71 (2011) 1008-1015.

[29] J. Gassan, A.K. Bledzki, Thermal degradation of flax and jute fibers, Journal of Applied Polymer Science 82 (2001) 1417-1422.

[30] P.R. Hornsby, E. Hinrichsen, K. Tarverdi, Preparation and properties of polypropylene composites reinforced with wheat and flax straw fibres: part I fibre characterization, Journal of Materials Science 32 (1997) 443-449. 